\title{
Effects of gelling agents and sugar substitutes on the quality characteristics of carrot jelly
}

\author{
Jong Jin Park, Ibukunoluwa Fola Olawuyi, Gwang Deok Park, Won Young Lee* \\ School of Food Science and Biotechnology, Kyungpook National University, Daegu 41566, Korea
}

\begin{abstract}
In this study, the quality characteristics of carrot jellies prepared using different gelling agents (gelatin and carrageenan) and sugar substitutes (sucrose, sorbitol, and xylitol) were evaluated. The effects of gelling agents and sugar substitutes on the $\mathrm{pH},{ }^{\circ}$ Brix, color, syneresis, melting-down rate, textural properties, and sensory acceptance of the jellies were determined. Textural analysis indicated that gelatin-based jellies containing sugar substitutes sorbitol and xylitol showed higher hardness than that of gelatin-based jelly containing sucrose. Among all samples, jellies containing $1.5 \%(\mathrm{w} / \mathrm{v})$ carrageenan showed higher hardness, cohesiveness, and chewiness than those of other jellies, regardless of the sugar substitute. The $\mathrm{pH}$ and ${ }^{\circ} \mathrm{Brix}$ of carrageenan-based jellies were lower than those of gelatin-based jellies. The instrumental color values of jellies containing gelatin decreased with an increase in gelatin concentration. Gelatin-based jellies ( 3 and $5 \%$ ) containing sucrose and gelatin-based jelly (5\%) containing xylitol showed lower syneresis than that in other jellies. Jelly containing $5 \%$ gelatin content had a lower melting-down rate compared with that containing $3 \%$ gelatin content. Moreover, carrageenan-based jellies showed a lower melting-down rate than that of gelatin-based jellies Carrot jellies containing 3\% gelatin and xylitol showed a high score for sensory acceptance. Based on the results, the utilization of $3 \%$ gelatin and xylitol as a sugar substitute is suitable for producing carrot jellies showing high sensory preference and stability.
\end{abstract}

Key words : carrot, jelly, gelling agent, sugar substitute

\section{Introduction}

Jelly is a semi-solid dessert, which is popular among individuals of all age groups because of its sweet taste and unique texture (Yoon and Shin, 2018). Currently, extracts or juices derived from natural products are incorporated into jelly formulations for various purposes, such as for substituting synthetic colorants, increasing nutritional value, and increasing bioactivity (Kang et al., 2017; Lee et al., 2020). Carrot (Daucus carota L.) is a root vegetable cultivated worldwide. Carrot is considered an important vegetable because of its high nutritional value owing to high vitamin, bioactive pigment, and fiber contents (Arscott and
Tanumihardjo, 2010), and is used as a functional ingredient in the production of carrot jelly (Kang et al., 2017; Nho et al., 2013). Typically, jelly is prepared using gelling agents (such as carrageenan and gelatin), sweeteners (such as sugar), and additives (coloring and flavoring agents). Sugar and gelling agents represent the main ingredients required for jelly production, and their variation in the jelly formulation is expected to influence its quality (Lee et al., 2008). However, the quality characteristics of carrot jellies associated with different gelling agents and sugars have not been studied.

Sugar plays important roles in the food system as a sweetener, in structure formation, and in reduction of water

\footnotetext{
*Corresponding author. E-mail : wonyoung@knu.ac.kr, Phone : +82-53-950-7763, Fax : +82-53-950-7762

Received 07 May 2021; Revised 17 June 2021; Accepted 02 July 2021.

Copyright (c) The Korean Society of Food Preservation.

This is an Open Access article distributed under the terms of the Creative Commons Attribution Non-Commercial License (http://creativecommons.org/licenses/by-nc/4.0) which permits unrestricted non-commercial use, distribution, and reproduction in any medium, provided the original work is properly cited.
} 
activity (Sandrou and Arvanitoyannis, 2000). However, the excessive intake of sugar can lead to development of metabolic disorders such as obesity and elevated blood pressure (Ahmad et al., 2020). Jelly contains large amounts of sugar, and previous studies have attempted to reduce or replace sugar in jelly products using fruit powder (Hwang et al., 2018), sugar substitutes (Riedel et al., 2015), and gelling agent mixtures (Khouryieh et al., 2005). Among these methods, sugar substitution is preferable in jelly production (Kim et al., 2008). Sugar substitutes represent sweetening agents that have sufficient sweetening capacity without an unpleasant aftertaste, and are non-carcinogenic and non-mutagenic (Gupta, 2018). Sugar substitutes are often used for therapeutic purposes, including assisting weight loss, dental care, and for managing diabetes mellitus and reactive hypoglycemia (Tandel, 2011). Moreover, sugar derivative sweeteners have been used to produce jellies that do not adversely affect consumer preferences (Choi et al., 2009).

Gelling agents, such as gelatin, pectin, agar, starch, carrageenan, and gellan gum represent food additives used to stabilize jelly (Banerjee and Bhattacharya, 2012). Gelatin is a biopolymeric protein derived from animal collagen, which has a reasonable cost and substantial production yield (Baziwane and He, 2003; Dai et al., 2018). Gelatin forms a stable three-dimensional gel by stabilizing hydrogen bonds with water molecules (Baziwane and $\mathrm{He}, 2003$ ), and a semi-solid colloidal gel after heating and cooling (Banerjee and Bhattacharya, 2012). Carrageenan is a natural polysaccharide obtained from edible red algae (Necas and Bartosikova, 2013). Carrageenan forms helical gels in the presence of a suitable cation (Banerjee and Bhattacharya, 2012). The gelation of carrageenan involves a coil-to-helix transition, followed by the subsequent aggregation of helices (Campo et al., 2009). Owing to their gelling properties, these materials are widely used as gelling agents in jelly production. Furthermore, the characteristics of gelling agents (for example, critical minimum concentration and molar mass/degree of polymerization) and other conditions for gel formation (for example, presence of sugar, $\mathrm{pH}$, ionic strength, and temperature) have a great influence on the textural properties, stability, and sensory characteristics of gel-based foods (Banerjee and Bhattacharya, 2012; Lee and Koh, 2017; Park et al., 2018). The effects of gelling agents, including gelatin and carrageenan, and sugars, including sucrose (SUC), sorbitol (SOR), and xylitol (XYL), on the characteristics of carrot jelly have not been investigated. Therefore, carrot jellies were produced in this study by varying these gelling agents and sugar substitutes. In addition, the quality characteristics of carrot jellies were measured to determine the optimal gelling agent and sugar.

\section{Materials and methods}

\section{Materials}

Carrots were purchased from a farm in Jeju Island, South Korea, and stored at $4^{\circ} \mathrm{C}$ until use. SUC (CJ Cheiljedang Co., Seoul, South Korea) and gelatin (Geltech, Busan, South Korea) were purchased from a local market. Carrageenan, XYL, and SOR were obtained from ES Food (Gunpo, South Korea).

\section{Jelly production}

The juice was obtained from peeled carrots using a Hurom juicer (HU-400; Hurom, Gimhae, Korea). The jelly formulation is listed in Table 1. Carrot jellies were produced using a previously described method with certain modifications (Park and Joo, 2006). Concentrations of gelling agents showing desirable jelly textural properties were selected based on a preliminary experiment. Gelling agents were dissolved separately in water in a $500 \mathrm{~mL}$ glass beaker while heating at $70^{\circ} \mathrm{C}$ for $2 \mathrm{~min}$. After the sugar/sugar substitutes were dissolved in the gelling solution for $2 \mathrm{~min}$, carrot juice and anhydrous citric acid (Jungbunzlauer Austria AG, Wulzeshofen, Austria) were added to the mixture. The mixture was then boiled at $90^{\circ} \mathrm{C}$ for $3 \mathrm{~min}$ and poured into a cubical mold $\left(2 \times 2 \times 2 \mathrm{~cm}^{3}\right)$. The samples were cooled to 2 $5^{\circ} \mathrm{C}$ for $30 \mathrm{~min}$ and placed in a refrigerator at $4^{\circ} \mathrm{C}$ for $2 \mathrm{~h}$.

\section{Texture analysis}

Texture profile analysis was performed using a rheometer (Compac-100; Scientific Co., Tokyo, Japan) containing a 5 $\mathrm{mm}$ diameter cylindrical probe at a table speed of 120 $\mathrm{mm} / \mathrm{min}$. The samples were cut into pieces $\left(1 \times 1 \times 1 \mathrm{~cm}^{3}\right)$ before analysis. Sample compression was set at $40 \%$. Measurements were repeated five times.

\section{Color measurement}

The surface color of jelly was measured with a chroma 
Table 1. Preparation of carrot jelly

\begin{tabular}{|c|c|c|c|c|}
\hline & \multicolumn{2}{|c|}{ Carrageenan } & \multicolumn{2}{|c|}{ Gelatin } \\
\hline & $1 \%$ & $1.5 \%$ & $3 \%$ & $5 \%$ \\
\hline Carrot juice (mL) & 180 & 180 & 180 & 180 \\
\hline Water $(\mathrm{mL})$ & 292.2 & 289.2 & 280.2 & 268.2 \\
\hline Gelling agent (g) & 6 & 9 & 18 & 30 \\
\hline Sugar $(g)$ & 120 & 120 & 120 & 120 \\
\hline Citric acid (g) & 1.8 & 1.8 & 1.8 & 1.8 \\
\hline Total amount (g) & 600 & 600 & 600 & 600 \\
\hline
\end{tabular}

meter (CR-300, Minolta Co., Osaka, Japan). The measurement was repeated five times. The results were expressed as $\mathrm{L}$ (lightness), a (redness-greenness), and b (yellowness-blueness).

\section{$\mathrm{pH}$ and ${ }^{\circ}$ Brix measurements}

Jelly products $(2 \mathrm{~g})$ were homogenized in distilled water (18 mL) using a homogenizer (PT 1200; Kinematica AG, Luzern, Switzerland) and centrifuged using Labogene 1248R centrifuge (Labogene Co., Ltd, Daejeon, South Korea) at 4,000 rpm for $10 \mathrm{~min}$. The $\mathrm{pH}$ and ${ }^{\circ}$ Brix of the supernatant were measured using a pH meter (FEP20; Mettler-Toledo, Schwerzenbach, Zurich, Switzerland) and a refractometer (Pocket refractometer PAL-1; ATAGO, Tokyo, Japan), respectively. The measurements were performed in triplicate.

\section{Syneresis analysis}

Syneresis was analyzed as described previously (Nagasaka and Taneya, 1996) with certain modifications. A desiccator was conditioned at $75 \%$ relative humidity using saturated sodium chloride. The samples were placed on a 6 mesh sieve and stored in the desiccator at $4^{\circ} \mathrm{C}$ for $24 \mathrm{~h}$. Syneresis was calculated using the following equation:

Syneresis $(\%)=$

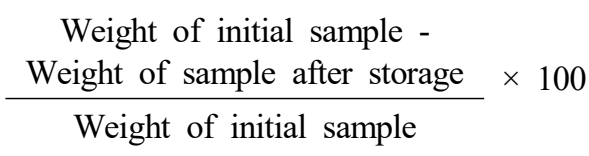

\section{Evaluation of melting-down rate}

The melting-down rate was determined as described by Kawamura (1989). A sol sample (50 g) was poured into a $100 \mathrm{~mL}$ beaker. The sample was cooled at room temperature for $30 \mathrm{~min}$ and then stored at $4^{\circ} \mathrm{C}$ for $2 \mathrm{~h}$. Subsequently, the gel sample was heated in a water bath (GO-90W; Jeio Tech, Daejeon, South Korea) at $30^{\circ} \mathrm{C}$ and $40^{\circ} \mathrm{C}$ for $10 \mathrm{~min}$. The sample was placed on a 6 mesh sieve for $30 \mathrm{~s}$, and the separated gel weights were measured. The melting-down rate was calculated as

$$
\begin{gathered}
\text { Syneresis }(\%)= \\
\frac{\begin{array}{c}
\text { Weight of initial sample }- \\
\text { Weight of sample after storage }
\end{array}}{\text { Weight of initial sample }} \times 100
\end{gathered}
$$

\section{Sensory evaluation}

Sensory evaluations were conducted to assess the preference of jelly according to the types of gelling agents and sugar. The sensory test was performed by a group of 25 semi-trained students. They evaluated the color, flavor, taste, texture, and overall acceptance on a seven-point hedonic scale (from 1 extremely bad to 7=extremely good). The sensory test was approved by Kyungpook National University Industry Foundation (Approval No: 2021-0018).

\section{Statistical analysis}

All experiments were performed in a minimum of triplicate. Duncan's test $(\mathrm{p}<0.05)$ was performed using the IBM SPSS Statistics (v.25, SPSS Inc., Chicago, IL, USA). Significance was set at $\mathrm{p}<0.05$.

\section{Results and discussion}

\section{Texture analysis}

The textural properties of carrot jellies containing 
different gelling agents and sugars are shown in Fig. 1. Gelatin-based jellies containing SOR and XYL sugar substitutes showed a higher hardness than that of gelatinbased jellies containing SUC (Fig. 1A). However, increasing the gelatin concentration from $3 \%$ to $5 \%(\mathrm{w} / \mathrm{v})$ did not significantly affect the hardness of gelatin-based jellies $(p<0.05)$. The hardness of carrageenan-based jellies significantly increased with an increase in carrageenan concentration, regardless of the sugar substitute used $(p<0.05)$. Similar results were obtained by $\mathrm{Oh}$ et al. (2013). The effect of sugar on hardness varied according to the carrageenan concentration used in jellies. When the carrageenan concentration used was $1 \%$, the hardness of jelly containing SOR was higher than that of jellies containing other sugars. In contrast, $1.5 \%$ carrageenan-based jelly containing SUC showed higher hardness than that of carrageenan jellies containing other sugar substitutes.

The cohesiveness of gelatin-based jellies was not significantly affected by varying gelatin concentrations or sugar substitutes (Fig. 1B). In contrast, the cohesiveness of carrageenan-based jellies increased with an increase in carrageenan concentration. Jellies containing $1 \%$ carrageenan except for that containing $1 \%$ carrageenan and sucrose
(C1SUC) showed lower cohesiveness than that of gelatinbased jellies, whereas jellies containing $1.5 \%$ carrageenan showed higher cohesiveness than that of gelatin-based jellies.

The springiness of gelatin-based jellies was not affected by varying gelatin concentrations or sugar substitutes. However, the springiness of carrageenan-based jellies containing SOR or XYL significantly increased with an increase in carrageenan concentration $(\mathrm{p}<0.05)$ (Fig. 1C).

The chewiness of carrot jellies increased with an increase in the concentration of gelling agent (Fig. 1D), and the effect was more noticeable in carrageenan-based jellies (concentration ranging from 1-1.5\%). Among all samples, jellies containing $1.5 \%$ carrageenan showed high chewiness. Variations in chewiness were also observed in jellies based on sugar substitute used. Overall, carrot jelly prepared using $1.5 \%$ carrageenan and sucrose (C1.5SUC) showed considerably high chewiness $(\mathrm{p}<0.05)$.

A previous study reported that the gelation of carrageenan is affected by the -OH groups of sugars and sugar alcohols (including SOR and XYL) (Stenner et al., 2016). Shimizu and Matubayasi (2014) similarly reported that sugar alcohols play an important role in the gelation capacity of gelatin
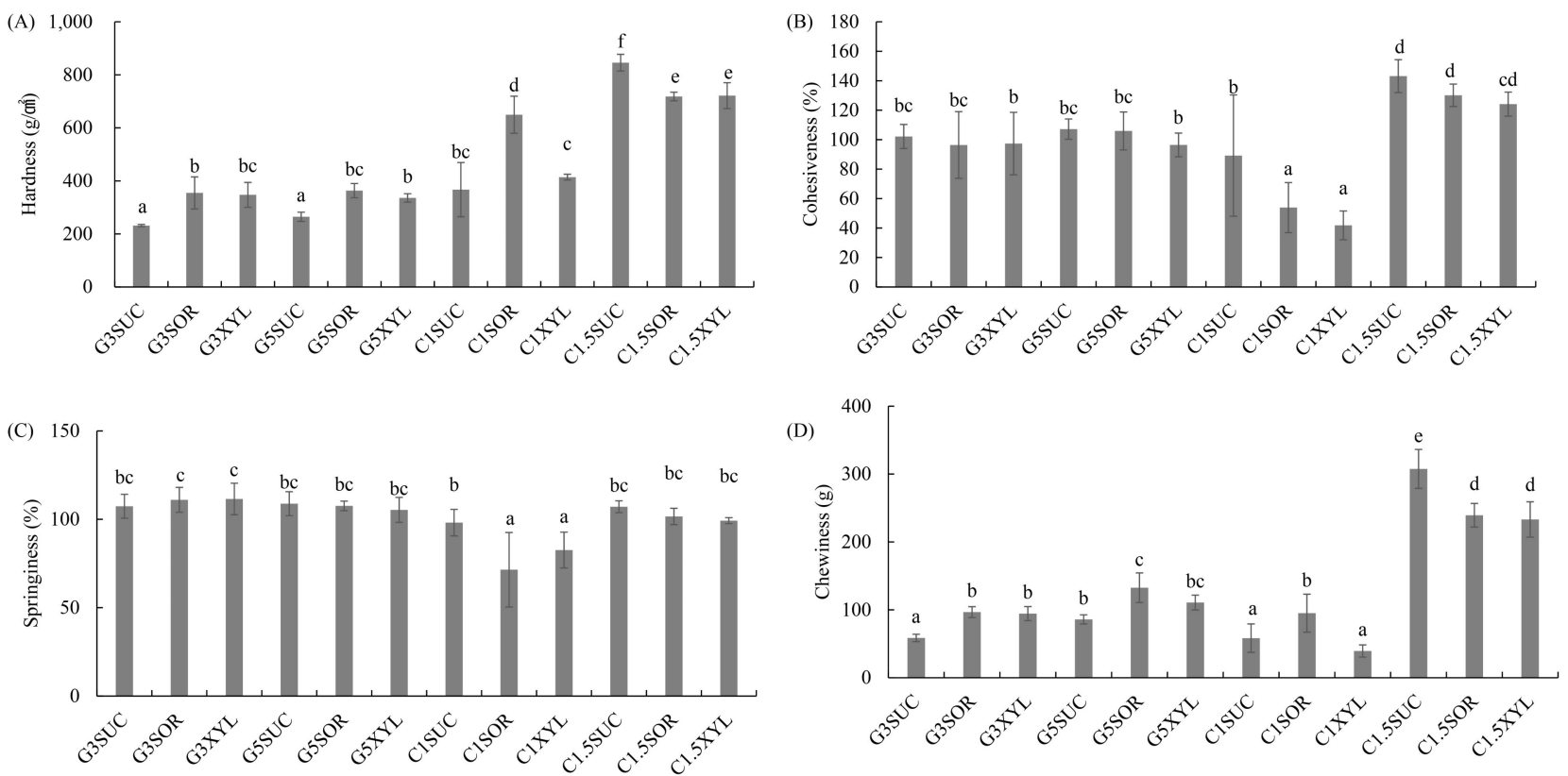

Fig. 1. The texture properties of carrot jellies according to gelling agents and sugar types.

Abbreviations: $3 \%$ gelatin (G3), 5\% gelatin (G5), 1\% carrageenan (C1), 1.5\% carrageenan (C1.5), sucrose (SUC), sobitol (SOR), and xylitol (XYL). Means with different letters are significantly different per Duncan's test $(p<0.05)$. 
because sugar alcohol represents the main mediator of gelation, and it can also influence the structure of water molecules surrounding the biopolymer. Therefore, the different textural properties of jellies containing different sugars and sugar alcohols may be attributed to the different characteristics of the sugars and sugar alcohols.

\section{Color}

The colors of the carrot jellies are presented in Table 2. Overall, the L, a, and b values of jellies containing gelatin decreased with an increase in gelatin concentration. Jellies containing gelatin $(3 \%)$ and either SOR (G3SOR) or XYL (G3XYL) showed higher L, a, and b values than those of jelly containing gelatin (3\%) and SUR (G3SUC); in contrast, jellies containing gelatin (5\%) and either SUC (G5SUC) or XYL (G5XYL) showed higher L and b values than jelly containing gelatin (5\%) and SOR (G5SOR). There were no significant differences in the color of jellies containing $1 \%$ carrageenan and different sugar substitutes $(p<0.05)$. However, when carrageenan concentration was $1.5 \%$, the color values of jelly containing carrageenan $(1.5 \%)$ and SOR (C1.5SOR) were lower than those of jellies containing carrageenan (1.5\%) and either SUC (C1.5SUC) or XYL (C1.5XYL). Similarly, a previous study reported that the color values of carrageenan-based jellies are affected based on the sugar substitute present (Lee et al., 2008). The color change may be attributed to pigment destruction or non-enzymatic browning during heating (Samakradhamrongthai and Jannu, 2021). Considering the identical conditions of thermal treatment used during jelly production, different effects of sugar/sugar alcohol on non-enzymatic browning may represent the main reason for the observed color differences (Naknaen and Itthisoponkul, 2015). During thermal treatment, the degradation of sucrose into glucose and fructose might have promoted the Maillard reaction. In contrast, the Maillard reaction occurs to a relatively lower extent in sugar alcohol than that in sucrose (Naknaen and Itthisoponkul, 2015). In addition, other factors, such as nutrients present in carrot juice and gelling agents, may have contributed to the difference in color of carrot jellies.

\section{Syneresis}

Syneresis involves the separation of a low-molecularweight liquid present on the surface of a gel during storage. Syneresis mainly occurs due to a lack of equilibrium in the structure owing to the formation of new bonds among

Table 2. The color values of carrot jellies according to gelling agents and sugar types

\begin{tabular}{|c|c|c|c|c|c|}
\hline Gelling agents & $\begin{array}{l}\text { Gelling agent } \\
\text { concentration } \\
(\% \mathrm{w} / \mathrm{v})\end{array}$ & Sugar types & $\begin{array}{c}\mathrm{L} \\
\text { (lightness) }\end{array}$ & $\begin{array}{c}\mathrm{a} \\
\text { (redness) }\end{array}$ & $\begin{array}{c}\mathrm{b} \\
\text { (yellowness) }\end{array}$ \\
\hline \multirow{6}{*}{ Gelatin } & \multirow{3}{*}{3} & $\mathrm{SUC}^{1)}$ & $39.12 \pm 1.40^{\mathrm{b} 2)}$ & $9.17 \pm 1.06^{\mathrm{bc}}$ & $24.21 \pm 0.83^{b c}$ \\
\hline & & SOR & $41.31 \pm 3.19^{c}$ & $13.75 \pm 3.48^{\mathrm{ef}}$ & $29.99 \pm 3.48^{\mathrm{def}}$ \\
\hline & & XYL & $42.64 \pm 2.23^{\mathrm{c}}$ & $15.05 \pm 3.42^{\mathrm{f}}$ & $30.96 \pm 2.71^{\mathrm{f}}$ \\
\hline & \multirow{3}{*}{5} & SUC & $38.35 \pm 1.12^{\mathrm{b}}$ & $7.13 \pm 0.26^{\mathrm{a}}$ & $22.23 \pm 0.73^{\mathrm{b}}$ \\
\hline & & SOR & $36.10 \pm 0.36^{\mathrm{a}}$ & $6.15 \pm 0.30^{\mathrm{a}}$ & $19.09 \pm 0.31^{\mathrm{a}}$ \\
\hline & & XYL & $38.79 \pm 0.65^{\mathrm{b}}$ & $7.77 \pm 0.23^{\mathrm{ab}}$ & $22.93 \pm 0.69^{b}$ \\
\hline \multirow{6}{*}{ Carrageenan } & \multirow{3}{*}{1} & SUC & $41.88 \pm 0.41^{\mathrm{c}}$ & $11.04 \pm 0.56^{\mathrm{cd}}$ & $28.58 \pm 0.63^{\mathrm{de}}$ \\
\hline & & SOR & $41.17 \pm 0.78^{\mathrm{c}}$ & $10.71 \pm 0.38^{\mathrm{cd}}$ & $28.61 \pm 2.05^{\mathrm{de}}$ \\
\hline & & XYL & $42.10 \pm 0.18^{\mathrm{c}}$ & $10.95 \pm 0.24^{\mathrm{cd}}$ & $30.52 \pm 0.55^{\mathrm{def}}$ \\
\hline & \multirow{3}{*}{1.5} & SUC & $41.93 \pm 0.27^{\mathrm{c}}$ & $12.58 \pm 0.13^{\mathrm{de}}$ & $28.41 \pm 0.39^{d}$ \\
\hline & & SOR & $38.77 \pm 0.99^{b}$ & $7.99 \pm 0.24^{\mathrm{ab}}$ & $26.00 \pm 0.45^{\mathrm{c}}$ \\
\hline & & XYL & $41.53 \pm 0.55^{\mathrm{c}}$ & $12.02 \pm 0.21^{\mathrm{de}}$ & $30.66 \pm 1.06^{\mathrm{ef}}$ \\
\hline
\end{tabular}

${ }^{1)}$ SUC, sucrose; SOR, sorbitol; XYL, xylitol.

${ }^{2)}$ Means in the same column with different letters are significantly different per Duncan's test $(\mathrm{p}<0.05)$. 
macromolecules (Toncheva et al., 1994). In jelly products, syneresis is influenced by the structure, quantity and type of the gelling agent, and the dry matter content (Toncheva et al., 1994).

The syneresis of jelly products containing different gelling agents and sugar substitutes is shown in Fig. 2. Jellies prepared using gelatin (2.02-4.94\%) showed lower syneresis than that in carrageenan-based jellies (3.07$8.10 \%$ ). This may be attributed to the immobilization of the aqueous phase by gelatin, subsequently reducing the extent of syneresis (Fiszman et al., 1999). When the gelling agents used were $3 \%$ gelatin and $1 \%$ carrageenan, XYL-based jellies G3XYL and C1XYL showed higher syneresis than jellies containing other sugars. In contrast, syneresis was reduced when the concentration of the gelling agent was increased to $5 \%$ gelatin and $1.5 \%$ carrageenan. Moreover, syneresis was the lowest in jellies containing SUC, followed by XYL and SOR, based on sugar substitute. When the sugar substitute was the same, syneresis decreased with an increase in gelling agent concentration, except for jellies containing SOR. Overall, the syneresis in G3SUC, G5SUC, and G5XYL jellies was significantly lower than that in other jellies $(\mathrm{p}<0.05)$. This may be due to differences in the hygroscopicity of the sugars. In addition, the water-holding capacity that varies with the composition of the jelly is known to affect syneresis (Farias et al., 2019; Sinthusamran et al., 2018).

\section{Melting-down rate}

The melting-down rate was measured to evaluate the stability of the gel structure of the jellies (Lee et al., 2008). The melting-down rate at $30^{\circ} \mathrm{C}$ of gelatin-based jellies was slightly decreased with an increase in gelatin concentration (Fig. 3A). The melting-down rate of $\mathrm{G} 3 \mathrm{SOR}$ at $30^{\circ} \mathrm{C}$ was significantly lower than that of G3SUC $(p<0.05)$. This may be due to the effect of SOR in combination with citric acid, which protects the triple-helix-like structure of gelatin (Dai et al., 2020). In contrast, the melting-down rate of gelatin-based jellies increased considerably at $40^{\circ} \mathrm{C}$ (Fig. $3 \mathrm{~A})$. When the temperature was $40^{\circ} \mathrm{C}$, the increase in gelatin concentration significantly reduced the melting-down rate; however, there was no difference in melting-down rates of jellies containing different sugar substitutes $(\mathrm{p}<0.05)$. When the temperature is higher than the melting point of gelatin, sugar substitutes may not affect the melting-down rate of jellies (Dai et al., 2020). The melting-down rate decreased as the concentration of carrageenan increased (Fig. 3B); however, there were no differences based on the melting temperature. Among the samples, C1.5SUC and C1.5XYL jellies showed low melting-down rates. Overall, gelatinbased jellies showed higher melting-down rates than those of carrageenan-based jellies. This may be due to the fact that carrageenan maintains a stable network structure at high temperatures, whereas gelatin changes to sol due to the loss of cross-linking (Kawamura, 1989). Similarly, Kim et al. (2020) reported that carrageenan-based jellies have a higher melting temperature than that of gelatin. The difference in the melting-down rate based on sugar/sugar alcohol content may be due to the gel-sol transition which varies with sugar and sugar alcohol substitutes (Shimizu and Matubayasi,

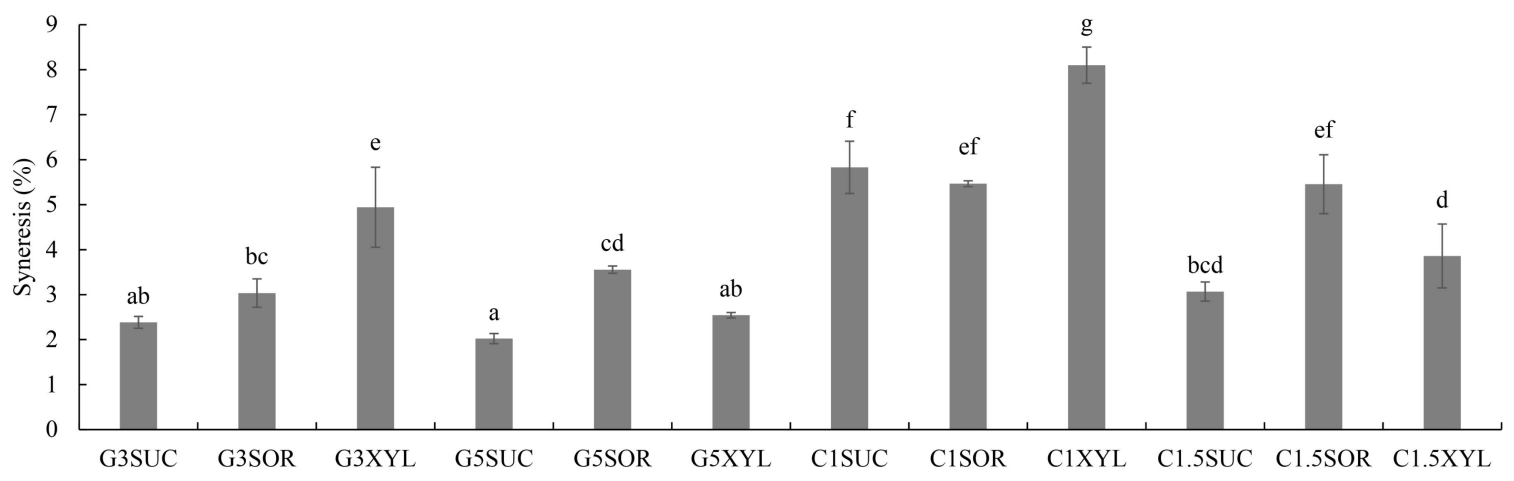

Fig. 2. The syneresis of carrot jellies according to gelling agents and sugar types.

Abbreviations: $3 \%$ gelatin (G3), 5\% gelatin (G5), 1\% carrageenan (C1), 1.5\% carrageenan (C1.5), sucrose (SUC), sobitol (SOR), and xylitol (XYL). Means with different letters are significantly different per Duncan's test $(\mathrm{p}<0.05)$. 

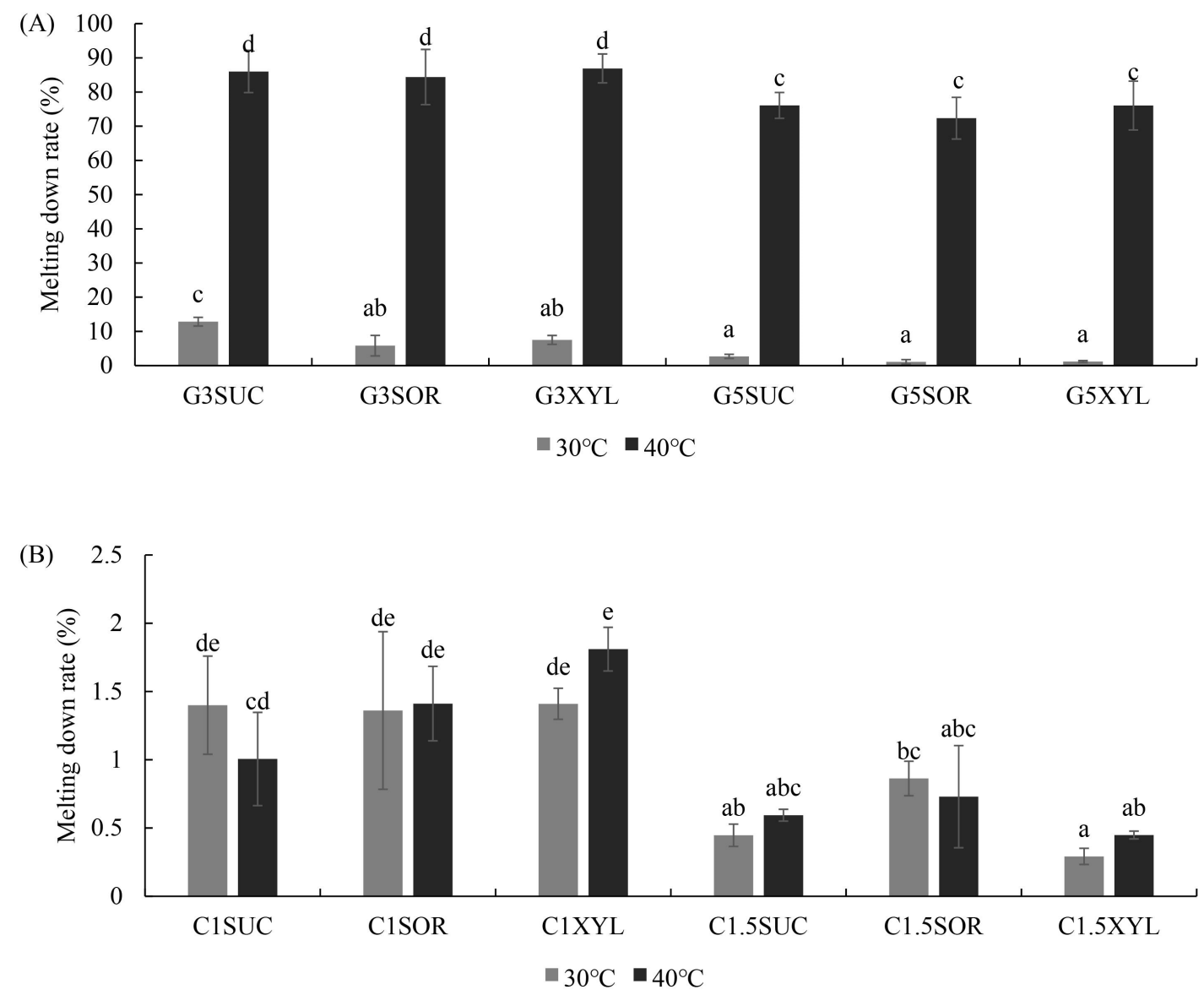

Fig. 3. The melting-down rate of carrot jellies according to gelling agents and sugar types.

Abbreviations: 3\% gelatin (G3), 5\% gelatin (G5), 1\% carrageenan (C1), 1.5\% carrageenan (C1.5), sucrose (SUC), sobitol (SOR), and xylitol (XYL). Means with different letters are significantly different per Duncan's test $(\mathrm{p}<0.05)$.

2014; Stenner et al., 2016).

\section{$\mathrm{pH}$ and ${ }^{\circ}$ Brix}

The $\mathrm{pH}$ and ${ }^{\circ}$ Brix values of the carrot jellies are shown in Table 3. Gelatin-based jellies showed a higher $\mathrm{pH}$ than that of carrageenan-based jellies. The $\mathrm{pH}$ increased with an increase in gelatin concentration. There was no correlation between carrageenan concentration and sugar substitute. When carrageenan was used as the gelling agent, XYLcontaining jellies showed a lower ${ }^{\circ}$ Brix than that of jellies containing SUC and SOR. The ${ }^{\circ}$ Brix value of carrageenanbased jellies (3.10-3.43 ${ }^{\circ}$ Brix) was lower than that of gelatin-based jellies (3.43-3.87 ${ }^{\circ}$ Brix). The gelling agent might have affected the refractive index during ${ }^{\circ}$ Brix measurements (Ryu et al., 2012).

\section{Sensory properties}

Sensory evaluations were performed to assess the preference for jelly products containing different gelling agents and sugar substitutes. Tables 4 and 5 present the sensory attributes of carrot jellies, including color, texture, flavor, taste, and overall acceptance. The sensory test was performed in two parts. First, a sensory test was performed for jellies varying in gelling agents used (Table 4). The G3SUC jelly showed the highest overall acceptance, taste, and texture. G5SUC jelly showed the lowest score for taste and flavor. Similar to G5SUC jelly, a low sensory score for jelly produced using a high gelatin concentration has been reported in a previous study (SG Park et al., 2014). The incorporation of a high concentration of gelatin might have affected the taste and flavor of the jelly. Carrageenan-based 
Table 3. The $\mathrm{pH}$ and ${ }^{\circ}$ Brix of carrot jellies according to gelling agents and sugar types

\begin{tabular}{|c|c|c|c|c|}
\hline Gelling agents & $\begin{array}{c}\text { Gelling agent concentration } \\
(\% \mathrm{w} / \mathrm{v})\end{array}$ & Sugar types & $\mathrm{pH}$ & ${ }^{\circ}$ Brix \\
\hline \multirow{6}{*}{ Gelatin } & \multirow{3}{*}{3} & $\mathrm{SUC}^{1)}$ & $4.04 \pm 0.02^{\mathrm{c} 2)}$ & $3.43 \pm 0.06^{\mathrm{c}}$ \\
\hline & & SOR & $4.03 \pm 0.03^{\mathrm{c}}$ & $3.77 \pm 0.06^{\mathrm{d}}$ \\
\hline & & XYL & $4.06 \pm 0.02^{\mathrm{c}}$ & $3.73 \pm 0.06^{\mathrm{d}}$ \\
\hline & \multirow{3}{*}{5} & SUC & $4.22 \pm 0.01^{\mathrm{d}}$ & $3.83 \pm 0.12^{\mathrm{d}}$ \\
\hline & & SOR & $4.21 \pm 0.01^{\mathrm{d}}$ & $3.83 \pm 0.06^{\mathrm{d}}$ \\
\hline & & XYL & $4.25 \pm 0.01^{\mathrm{e}}$ & $3.87 \pm 0.06^{\mathrm{d}}$ \\
\hline \multirow{6}{*}{ Carrageenan } & \multirow{3}{*}{1} & SUC & $3.76 \pm 0.01^{\mathrm{b}}$ & $3.43 \pm 0.21^{\mathrm{c}}$ \\
\hline & & SOR & $3.72 \pm 0.00^{\mathrm{a}}$ & $3.30 \pm 0.10^{\mathrm{bc}}$ \\
\hline & & XYL & $3.75 \pm 0.01^{\mathrm{b}}$ & $3.10 \pm 0.10^{\mathrm{a}}$ \\
\hline & \multirow{3}{*}{1.5} & SUC & $3.75 \pm 0.02^{\mathrm{b}}$ & $3.43 \pm 0.06^{\mathrm{c}}$ \\
\hline & & SOR & $3.74 \pm 0.00^{\mathrm{ab}}$ & $3.33 \pm 0.06^{\mathrm{bc}}$ \\
\hline & & XYL & $3.72 \pm 0.01^{\mathrm{a}}$ & $3.17 \pm 0.15^{\mathrm{ab}}$ \\
\hline
\end{tabular}

${ }^{1)}$ SUC, sucrose; SOR, sorbitol; XYL, xylitol.

${ }^{2)}$ Means in the same column with different letters are significantly different per Duncan's test $(\mathrm{p}<0.05)$.

Table 4. The sensory attributes of carrot jellies with sucrose according to gelling agents

\begin{tabular}{cccccc}
\hline & Color & Flavor & Taste & Texture & Overall \\
\hline C1SUC & $5.57 \pm 1.03^{\mathrm{a}}$ & $4.62 \pm 1.36^{\mathrm{a}}$ & $4.43 \pm 1.33^{\mathrm{ab}}$ & $3.48 \pm 1.72^{\mathrm{a}}$ & $4.10 \pm 1.51^{\mathrm{a}}$ \\
C1.5SUC & $5.71 \pm 0.96^{\mathrm{a}}$ & $4.52 \pm 1.44^{\mathrm{a}}$ & $4.67 \pm 1.24^{\mathrm{ab}}$ & $4.38 \pm 1.43^{\mathrm{ab}}$ & $4.29 \pm 1.27^{\mathrm{a}}$ \\
G3SUC & $5.52 \pm 1.17^{\mathrm{a}}$ & $4.43 \pm 1.43^{\mathrm{a}}$ & $5.05 \pm 1.16^{\mathrm{b}}$ & $5.38 \pm 1.16^{\mathrm{c}}$ & $5.19 \pm 0.93^{\mathrm{b}}$ \\
G5SUC & $5.24 \pm 1.37^{\mathrm{a}}$ & $4.05 \pm 1.40^{\mathrm{a}}$ & $4.14 \pm 1.62^{\mathrm{a}}$ & $4.57 \pm 1.63^{\mathrm{bc}}$ & $4.81 \pm 1.17^{\mathrm{ab}}$ \\
\hline
\end{tabular}

Abbreviations: $3 \%$ gelatin $(\mathrm{G} 3), 5 \%$ gelatin $(\mathrm{G} 5), 1 \%$ carrageenan $(\mathrm{C} 1), 1.5 \%$ carrageenan $(\mathrm{C} 1.5)$, and sucrose (SUC). Means in the same column with different letters are significantly different per Duncan's test $(p<0.05)$.

Table 5. The sensory attributes of carrot jellies with gelatin according to sugar types

\begin{tabular}{cccccc}
\hline & Color & Flavor & Taste & Texture & Overall \\
\hline G3SUC & $5.10 \pm 1.37^{\mathrm{a}}$ & $4.15 \pm 1.23^{\mathrm{a}}$ & $4.25 \pm 1.29^{\mathrm{ab}}$ & $4.70 \pm 1.66^{\mathrm{a}}$ & $4.73 \pm 1.22^{\mathrm{a}}$ \\
G3SOR & $4.95 \pm 1.54^{\mathrm{a}}$ & $4.00 \pm 1.52^{\mathrm{a}}$ & $3.65 \pm 1.69^{\mathrm{a}}$ & $4.25 \pm 1.52^{\mathrm{a}}$ & $4.41 \pm 1.55^{\mathrm{a}}$ \\
G3XYL & $5.50 \pm 1.19^{\mathrm{a}}$ & $4.10 \pm 1.33^{\mathrm{a}}$ & $4.55 \pm 1.64^{\mathrm{ab}}$ & $4.35 \pm 1.50^{\mathrm{a}}$ & $4.82 \pm 1.18^{\mathrm{a}}$ \\
\hline
\end{tabular}

Abbreviations: 3\% gelatin (G3), sucrose (SUC), sorbitol (SOR), and xylitol (XYL).

Means in the same column with different letters are significantly different per Duncan's test $(\mathrm{p}<0.05)$

jellies showed high scores for flavor and color; however, the texture and overall acceptance of carrageenan-based jellies were lower than those of gelatin-based jellies. Based on these results, G3SUC showed higher preferences for texture, taste, and overall acceptance. Therefore, 3\% gelatin was selected as the gelling agent for subsequent sensory evaluation.

The second sensory test was performed for jellies containing 3\% gelatin that varied in sugar substitutes (Table 5). Lee et al. (2008) reported that fruit jellies produced using 
sugar substitutes may be less preferable owing to differences in the sense of taste of individuals. However, in this study, G3XYL showed a higher score for taste than that of G3SUC. XYL shows an effect similar to that of sugar; therefore, the sweetness of XYL pairs well with that of carrot juice, which might have influenced its high score (Choi et al., 2009). The flavor scores of the jellies were not affected by sugar substitutes. G3SUC showed a slightly higher score for texture, followed by G3XYL and G3SOR. G3SUC had a lower hardness and chewiness than those of jellies containing other sugars (Fig. 1). Jellies containing $1.5 \%$ carrageenan had the highest hardness and chewiness values. However, these jellies showing high hardness and chewiness showed low sensory scores for texture and overall acceptability. These results indicate that the hardness of jelly corresponds to a low sensory preference. Jellies containing XYL showed a brighter color than that of jellies containing other sugars, which might have influenced their high scores in the sensory test (Table 2). G3SUC and G3XYL showed a slightly higher overall acceptance than that of G3SOR, but the difference was not significant $(p>0.05)$. Overall, the sensory results indicated that XYL may represent a suitable sugar substitute for jellies containing 3\% (w/v) gelatin.

\section{Conclusion}

This study confirmed that the properties of carrot jellies are substantially affected by the type and concentration of the gelling agent and the type of sugar substitute used. Jellies containing $1.5 \%(\mathrm{w} / \mathrm{v})$ carrageenan showed higher hardness, chewiness, and cohesiveness than those of other jellies. When gelatin was used as the gelling agent, jellies containing SOR and XYL sugar substitutes showed higher hardness and chewiness than those of SUC-containing jellies. Among the jellies, C1SUC and C1SOR showed the lowest springiness. The syneresis and melting-down rate of jellies decreased with an increase in gelling agent concentration. Syneresis was lower in gelatin-based jellies than that in carrageenan-based jellies. In particular, G3SUC, G5SUC, and G5XYL jellies showed the lowest syneresis. Carrageenan-based jellies showed lower melting-down rates than those of gelatin-based jellies. Sensory evaluation indicated that $5 \%(\mathrm{w} / \mathrm{v})$ gelatin-based jellies were associated with a low preference for taste and flavor. Moreover, carrageenan-based jellies showed a low score for overall acceptability and texture. Considering quality parameters and sensory attributes, $3 \%(\mathrm{w} / \mathrm{v})$ gelatin was suitable for use as a gelling agent for producing carrot jelly, based on findings of previous studies and this study. Moreover, XYL was the most preferred sugar substitute for producing carrot jelly.

\section{Conflict of interests}

The authors declare no potential conflict of interest.

\section{ORCID}

Jong Jin Park https://orcid.org/0000-0001-9815-4101

Won Young Lee https://orcid.org/0000-0001-5850-9692

\section{References}

Ahmad R, Mok A, Rangan AM, Louie JCY. Association of free sugar intake with blood pressure and obesity measures in Australian adults. Eur J Nutr, 59, 651-659 (2020)

Arscott SA, Tanumihardjo SA. Carrots of many colors provide basic nutrition and bioavailable phytochemicals acting as a functional food. Compr Rev Food Sci Food Saf, 9, 223-239 (2010)

Banerjee S, Bhattacharya S. Food gels: Gelling process and new applications. Crit Rev Food Sci Nutr, 52, 334-346 (2012)

Baziwane D, He Q. Gelatin: The paramount food additive. Food Rev Int, 19, 423-435 (2003)

Campo VL, Kawano DF, da Silva Jr DB, Carvalho I. Carrageenans: Biological properties, chemical modifications and structural analysis-A review. Carbohydr Polym, 77, 167-180 (2009)

Choi EJ, Lee MH, Oh MS. Quality characteristics of Jeju mandarin orange jellies with sugar derivative sweeteners for consumption by the elderly. J Korean Soc Food Cult, 24, 212-218 (2009)

Dai H, Li X, Du J, Ma L, Yu Y, Zhou H, Guo T, Zhang Y. Effect of interaction between sorbitol and gelatin on gelatin properties and its mechanism under different citric acid concentrations. Food Hydrocolloids, 101, 105557 (2020) 
Dai H, Ou S, Huang Y, Liu Z, Huang H. Enhanced swelling and multiple-responsive properties of gelatin/sodium alginate hydrogels by the addition of carboxymethyl cellulose isolated from pineapple peel. Cellulose, 25, 593-606 (2018)

Farias TRT, Schiassi MCEV, Pereira PAP, de Souza VR, Lago AMT, Borges SV, Queiroz F. Mixed Brazilian Cerrado fruits preserves without added sugar: The effect of bodying agents. Br Food J, 121, 1969-1981 (2019)

Fiszman, S, Lluch, M, Salvador, A. Effect of addition of gelatin on microstructure of acidic milk gels and yoghurt and on their rheological properties. Int Dairy J, 9, 895-901 (1999)

Gupta M. Sugar substitutes: Mechanism, availability, current use and safety concerns-an update. Open Access Maced J Med Sci, 6, 1894 (2018)

Hwang K, Kang S, Kim D, Lee H, Lee S, Choi Y, Jang E, Park S. Physicochemical characteristics of Yuza (Citrus junos Sieb ex Tanaka) powder added lowsugar jelly. J Chitin Chitosan, 23, 76-83 (2018)

Kang HS, Kim MJ, Rho JO, Choi HI, Han MR, Myung JH, Kim AJ. Quality characteristics of care food (jelly) prepared with wild carrot (Daucus carota L.) juice. J Korean Diet Assoc, 23, 337-349 (2017)

Kawamura F. The properties of gelatin gel and sol mixed with carrageenan (Part 1), effect of mixing ratio on the properties. J Cookery Sci Jpn, 22, 147-151 (1989)

Khouryieh HA, Aramouni FM, Herald TJ. Physical, chemical and sensory properties of sugarfree jelly. J Food Qual, 28, 179-190 (2005)

Kim MJ, Yoon SH, Jung MY, Choe EO. Effects of sugars and pectin on the quality characteristics of low sugar wild vine (Vitis coignetiea) jam. Korean J Food Cookery Sci, 24, 206-211 (2008)

Kim YM, Kim JM, Youn KS. Quality and textural properties of jelly prepared with different gelling agents. Korean J Food Preserv, 27, 566-573 (2020)

Lee EJ, Koh BK. Quality properties of rice Mook with the addition of gelling agents. Korean J Food Cookery Sci, 33, 87-93 (2017)

Lee ES, Lee YJ, Kim JH, Chun SS. Quality characteristics of jelly with lemon myrtle (Backhousia citriodora) extracts. Korean J Food Nutr, 33, 131-141 (2020)

Lee MH, Choi EJ, Oh MS. Quality characteristics of grape jellies with sugar derivative sweeteners for the elderly. J Korean Soc Food Cult, 23, 499-506 (2008)

Nagasaka K, Taneya Si. Analysis of syneresis rate of agar gel: Syneresis phenomena of foods. Part 1. J Jpn Soc Food Sci, 43, 1176-1182 (1996)

Naknaen P, Itthisoponkul T. Characteristics of cantaloupe jams as affected by substitution of sucrose with xylitol. Int J Fruit Sci, 15, 442-455 (2015)

Nho HJ, Jang SY, Park JJ, Yun HS, Park SM. Browning prevention of black carrot extract and the quality characteristics of jelly supplemented with black carrot extract. J Korean Soc Food Cult, 28, 293-302 (2013)

Oh HJ, Back JW, Lee JY, Oh YJ, Lim SB. Quality characteristics of jelly added with pressed kiwi (Actinidia chinensis var. 'Halla Gold') juice. Culi Sci Hos Res, 19, 110-120 (2013)

Park GY, Ra HN, Cho YS, Kim HY, Kim KM. Effects of addition of mixed gelling agent on quality of peach (Prunus persica L. Batsch) jelly. J Korean Soc Food Cult, 33, 458-463 (2018)

Park SG, Song TH, Kim DH, Kim GH, Jang KI. Quality properties of peach pudding added with Korean peach (Prunus persica L. Batsch) juice and gelatin. J Korean Soc Food Sci Nutr, 43, 265-272 (2014)

Park SH, Joo NM. Optimization of jelly with addition of Morinda citrifolia (Noni) by response surface methodology. Korean J Food Cookery Sci, 22, 1-11 (2006)

Riedel R, Bohme B, Rohm H. Development of formulations for reduced-sugar and sugar-free agar-based fruit jellies. Int J Food Sci Technol, 50, 1338-1344 (2015)

Ryu J, Jung J, Lee S, Ko S. Comparison of physicochemical properties of agar and gelatin gel with uniform hardness. Food Eng Prog, 16, 14-19 (2012)

Samakradhamrongthai RS, Jannu T. Effect of stevia, xylitol, and corn syrup in the development of velvet tamarind (Dialium indum L.) chewy candy. Food Chem, 352, 129353 (2021)

Sandrou D, Arvanitoyannis I. Low-fat/calorie foods: Current state and perspectives. Crit Rev Food Sci Nutr, 40, 427-447 (2000)

Shimizu S, Matubayasi N. Gelation: The role of sugars and polyols on gelatin and agarose. J Phys Chem B, 118, 13210-13216 (2014)

Sinthusamran S, Benjakul S, Hemar Y. Physical and sensory 
properties of gelatin from seabass (Lates calcarifer) as affected by agar and $\kappa$ carrageenan. J Texture Stud, 49, 47-55 (2018)

Stenner R, Matubayasi N, Shimizu S. Gelation of carrageenan: Effects of sugars and polyols. Food Hydrocolloids, 54, 284-292 (2016)

Tandel KR. Sugar substitutes: Health controversy over perceived benefits. J Pharmacol Pharmacother, 2,
236-243 (2011)

Toncheva G, Hadjikinov D, Panchev I. Investigation of syneresis of agar jellies with sorbitol. Food Chem, 49, 29-31 (1994)

Yoon JA, Shin KO. Studies of Tarak, a Korean traditional fermented milk product. J Dairy Sci Biotechnol, 36, 26-31 (2018) 